\title{
Estimation of Entropy for Weibull Distribution Based on Record Values
}

\author{
Manoj Chacko*, P. S. Asha \\ Department of Statistics, University of Kerala, Trivandrum, 695581, India
}

\section{ARTICLE INFO}

\section{Article History}

Received 05 Apr 2019

Accepted 14 Dec 2020

\section{Keywords:}

Entropy

record values

Bayes estimators

MCMC method

2000 Mathematics Subject

Classification: 94A17, 62F10, 62F15

\section{ABSTRACT}

In this paper, estimation of entropy for Weibull distribution based on record values is considered. Maximum likelihood estimation and Bayes estimation for Shannon entropy and Renyi entropy have been considered based on record values. Bayes estimators are obtained using Markov Chain Monte Carlo (MCMC) method. A simulation study is performed to find the performance of the estimators developed in this paper. The inferential procedures developed in this paper have also been illustrated using real data.

(C) 2021 The Authors. Published by Atlantis Press B.V. This is an open access article distributed under the CC BY-NC 4.0 license (http://creativecommons.org/licenses/by-nc/4.0/).

\section{INTRODUCTION}

Let $\left\{X_{i}, i \geq 1\right\}$ be a sequence of independent and identically distributed (iid) random variables having an absolutely continuous cumulative distribution function (cdf) $F(x)$ and probability density function (pdf) $f(x)$. An observation $X_{j}$ is called an upper record if $X_{j}>X_{i}$ for every $i<j$. An analogous definition deals with lower record values. In a number of situations, only observations that exceed or only those that fall below the current extreme value are recorded. Examples include meteorology, hydrology, athletic events, mining, etc. Interest in records has increased steadily over the years since Chandler [1] formulation. Useful surveys are given in Ahsanullah [2] and Arnold et al. [3]. Let $R_{i}, i=1,2, \ldots, n$ be the first $n$ upper record values arising from a distribution with cdf $F(x)$ and pdf $f(x)$. Let $\mathbf{D}_{\mathbf{n}}=\left(R_{1}, R_{2}, \cdots, R_{n}\right)$. Then the joint density function of $\mathbf{D}_{\mathbf{n}}$ is given by

$$
f\left(r_{1}, r_{2}, \ldots, r_{n}\right)=f\left(r_{n}\right) \prod_{i=1}^{n-1} \frac{f\left(r_{i}\right)}{1-F\left(r_{i}\right)},-\infty<r_{1}<r_{2}<\ldots<r_{n}<\infty .
$$

In reliability theory, record values are used for statistical modeling, shock models and they are closely connected with the occurrence times of a corresponding nonhomogeneous Poisson process, with some minimal repair scheme (see, Kamps [4]). Estimation of parameters using record values and prediction of future record values have been studied by several authors, for details see Balakrishnan and Chan [5], Raqab [6] and Sultan et al. [7]. Bayesian estimation and prediction for some life distributions based on record values have been considered by Ahmadi and Doostparast [8]. Seo et al. [9] developed an entropy estimation method for upper record values from the generalized halflogistic distribution.

Entropy measures the uncertainty associated with a random variable. Let $X$ be a random variable having an absolutely continuous cdf $F(x)$ and $\operatorname{pdf} f(x)$ then the entropy introduced by Shannon [10], called Shannon entropy is defined by

$$
H(f)=-\int_{-\infty}^{\infty} f(x) \log (f(x)) d x
$$

Several generalized entropy measures have been proposed in the literature to measure the uncertainty of a probability distribution since the seminal work of Shannon [10]. Among these, one of the most important and applicable measures is Renyi entropy proposed by Renyi [11].

*Corresponding author. Email: manojchacko02@gmail.com 
For a random variable $X$ with pdf $f(x)$ the Renyi entropy is given by

$$
H_{\alpha}(f)=\frac{1}{1-\alpha} \log \int_{-\infty}^{\infty} f(x)^{\alpha} d x ; \quad \alpha(\neq 1)>0 .
$$

Several authors have discussed the problem of estimation for entropy functions for different distributions. Kang et al. [12] derived estimators of entropy for a double exponential distribution based on multiply Type II censored samples. Cho et al. [13] derived estimators for the entropy function of a Rayleigh distribution based on doubly-generalized Type II hybrid censored samples. Baratpour et al. [14] developed the entropy of upper record values and provided several upper and lower bounds for this entropy by using the hazard rate function. Chacko and Asha [15] considered the problem of estimation of entropy for generalized exponential distribution using lower record values.

In this paper we consider the problem of estimation for entropy of a two parameter Weibull distribution with cdf given by

$$
F(x \mid \beta, \lambda)=1-e^{-\lambda x^{\beta}}
$$

The pdf corresponding to the above cdf is

$$
f(x \mid \beta, \lambda)=\beta \lambda x^{\beta-1} e^{-\lambda x^{\beta}}
$$

The Weibull distribution has wide applications in broad areas including reliability engineering, survival analysis, hydrology, meteorology and insurance (see, Murthy et al. [16]). Also, parametric inference of Weibull distribution based on record data is of special interest, because the Weibull distribution arises naturally from the extreme value theorem and thus it has a meaningful physical interpretation in many real applications (see, Ye et al. [17]). In various engineering applications such as independent component analysis, image analysis, genetic analysis and time delay estimation it is useful to estimate the entropy of a system or process given some observations. If the observations available are only in the form of record values and the parent population follows Weibull distribution then the inferential procedure developed in this work can be utilized to estimate the entropies of the system or process.

The Shanon entropy for the Weibull distribution with cdf given in (4) is given by

$$
H(\lambda, \beta)=v\left(1-\frac{1}{\beta}\right)-\log \left(\beta \lambda^{1 / \beta}\right)+1
$$

where $v$ is the Euler constant. The Renyi entropy for the Weibull distribution with cdf given in (4) is given by

$$
\begin{aligned}
H_{\alpha}(\lambda, \beta)= & \frac{1}{1-\alpha}\left[(\alpha-1) \log \beta+\alpha \log \lambda+\log \left(\Gamma\left(\alpha\left(1-\frac{1}{\beta}\right)+\frac{1}{\beta}\right)\right)\right. \\
& \left.\left(\alpha\left(1-\frac{1}{\beta}\right)+\frac{1}{\beta}\right) \log (\alpha \lambda)\right] .
\end{aligned}
$$

Cramer and Bagh [18] discussed the entropy in the Weibull distribution for progressive censoring. Cho et al. [19] obtained estimators for the entropy function of a Weibull distribution based on generalized Type II hybrid censored samples.

In this paper, we consider the estimation of entropies for Weibull distribution based on upper record values. In Section 2 we obtain the maximum likelihood estimators (MLEs) for Shannon entropy and Renyi entropy using upper record values. In Section 3, we consider the Bayes estimation of entropies using MCMC method. Section 4 is devoted to some simulation studies. In Section 5, a real data is used for illustration. Finally in Section 6 some concluding remarks.

\section{MAXIMUM LIKELIHOOD ESTIMATION}

In this section, we obtain the MLEs of entropies for the two parameter Weibull distribution using upper record values. Let $R_{i}, i=1,2, \ldots, n$ be the first $n$ upper record values arising from Weibull distribution with cdf given in (4). Let $\mathbf{D}_{\mathbf{n}}=\left(R_{1}, R_{2}, \cdots, R_{n}\right)$. Then from $(1)$ the likelihood function is given by

$$
L\left(\lambda, \beta \mid \mathbf{d}_{\mathbf{n}}\right)=(\lambda \beta)^{n} e^{-\lambda r_{n}^{\beta}} \prod_{i=1}^{n} r_{i}^{\beta-1},
$$

where $\mathbf{d}_{\mathbf{n}}=\left(r_{1}, r_{2}, \cdots, r_{n}\right)$. The log likelihood function is given by

$$
\log L\left(\lambda, \beta \mid \mathbf{d}_{\mathbf{n}}\right)=n \log (\lambda \beta)-\lambda r_{n}^{\beta}+(\beta-1) \sum_{i=1}^{n} \log \left(r_{i}\right) .
$$


Then

$$
\frac{\partial \log L}{\partial \beta}=\frac{n}{\beta}-\lambda r_{n}^{\beta} \log \left(r_{n}\right)+\sum_{i=1}^{n} \log \left(r_{i}\right)
$$

and

$$
\frac{\partial \log L}{\partial \lambda}=\frac{n}{\lambda}-r_{n}^{\beta}
$$

Then the corresponding normal equations are

$$
\frac{n}{\beta}-\lambda r_{n}^{\beta} \log \left(r_{n}\right)+\sum_{i=1}^{n} \log \left(r_{i}\right)=0
$$

and

$$
\frac{n}{\lambda}-r_{n}^{\beta}=0
$$

From (11), we get

$$
\lambda=\frac{n}{r_{n}^{\beta}} .
$$

By putting the value of $\lambda$ in (10) we get

$$
\frac{n}{\beta}-n \log \left(r_{n}\right)+\sum_{i=1}^{n} \log \left(r_{i}\right)=0 .
$$

Therefore, the MLE of $\beta$ is given by

$$
\hat{\beta}=\frac{n}{n \log \left(r_{n}\right)-\sum_{i=1}^{n} \log \left(r_{i}\right)} .
$$

Thus the MLE of $\lambda$ is obtained as

$$
\hat{\lambda}=\frac{n}{r_{n}^{\hat{\beta}}} .
$$

Then by invariant property of MLE, the MLE of Shannon entropy for Weibull distribution based on upper record values is given by

$$
\hat{H}_{M L}=v\left(1-\frac{1}{\hat{\beta}}\right)-\log \left(\hat{\beta} \hat{\lambda}^{1 / \hat{\beta}}\right)+1
$$

Similarly MLE of Renyi entropy for Weibull distribution based on upper record values is given by

$$
\begin{aligned}
\hat{H}_{(M L)}(\alpha)= & \frac{1}{1-\alpha}\left[(\alpha-1) \log \hat{\beta}+\alpha \log \hat{\lambda}+\log \left(\Gamma\left(\alpha\left(1-\frac{1}{\hat{\beta}}\right)+\frac{1}{\hat{\beta}}\right)\right)\right. \\
& \left.-\left(\alpha\left(1-\frac{1}{\hat{\beta}}\right)+\frac{1}{\hat{\beta}}\right) \log (\alpha \hat{\lambda})\right] .
\end{aligned}
$$




\section{BAYESIAN ESTIMATION}

In this section, we consider Bayesian estimation of entropy for the two parameter Weibull distribution under symmetric as well as asymmetric loss functions. A symmetric loss function is the squared error loss (SEL) function which is defined as

$$
L_{1}(d(\mu), \hat{d}(\mu))=(\hat{d}(\mu)-d(\mu))^{2}
$$

where $\hat{d}(\mu)$ is an estimate of $d(\mu)$. The Bayes estimate of $\mu$ under $L_{1}$ is the posterior mean of $\mu$. An asymmetric loss function is the LINEX loss (LL) function which is defined as

$$
L_{2}(d(\mu), \hat{d}(\mu))=e^{h(\hat{d}(\mu)-d(\mu))}-h(\hat{d}(\mu)-d(\mu))-1, h \neq 0
$$

The Bayes estimate of $d(\mu)$ for the loss function $L_{2}$ can be obtained as

$$
\hat{d}_{L B}(\mu)=-\frac{1}{h} \log \left\{E_{\mu}\left(e^{-h \mu} \mid \underline{x}\right)\right\}
$$

provided $E_{\mu}(\cdot)$ exists. Another asymmetric loss function is the general entropy loss (EL) function given by

$$
L_{3}(d(\mu), \hat{d}(\mu))=\left(\frac{\hat{d}(\mu)}{d(\mu)}\right)^{q}-q \log \left(\frac{\hat{d}(\mu)}{d(\mu)}\right)-1, q \neq 0
$$

In this case Bayes estimate of $d(\mu)$ is obtained as

$$
\hat{d}_{E B}(\mu)=\left(E_{\mu}\left(\mu^{-q} \mid \underline{x}\right)\right)^{-\frac{1}{q}}
$$

Let $R_{i}, i=1,2, \ldots, n$ be the first $n$ upper record values arising from Weibull distribution with pdf given in (5). Then the likelihood function is given by

$$
L\left(\lambda, \beta \mid \mathbf{d}_{\mathbf{n}}\right)=(\lambda \beta)^{n} e^{-\lambda r_{n}^{\beta}} \prod_{i=1}^{n} r_{i}^{\beta-1}
$$

where $\mathbf{d}_{\mathbf{n}}=\left(r_{1}, r_{2}, \cdots, r_{n}\right)$. Assume that the prior distributions of $\beta$ and $\lambda$ follow independent gamma distributions with density functions respectively given by

$$
\pi_{1}(\beta \mid a, b)=\frac{b^{a}}{\Gamma a} \beta^{a-1} e^{-b \beta} ; a>, b>0
$$

and

$$
\pi_{2}(\lambda \mid c, d)=\frac{d^{c}}{\Gamma c} \lambda^{c-1} e^{-d \lambda} ; c>0, d>0
$$

Thus the joint prior distribution of $\lambda$ and $\beta$ is given by

$$
\pi(\lambda, \beta)=\frac{b^{a} d^{c}}{\Gamma a \Gamma c} \beta^{a-1} \lambda^{c-1} e^{-b \beta} e^{-d \lambda} .
$$

Then the joint posterior density of $\lambda$ and $\beta$ given $\mathbf{D}_{\mathbf{n}}=\mathbf{d}_{\mathbf{n}}$ can be written as

$$
\pi^{*}\left(\lambda, \beta \mid \mathbf{d}_{\mathbf{n}}\right)=\frac{L\left(\lambda, \beta \mid \mathbf{d}_{\mathbf{n}}\right) \pi(\lambda, \beta)}{\iint L\left(\lambda, \beta \mid \mathbf{d}_{\mathbf{n}}\right) \pi(\lambda, \beta) d \lambda d \beta} .
$$

Therefore the Bayes estimate of any function $g(\beta, \lambda)$ of $\beta$ and $\lambda$ under SEL, LL and EL are respectively given by

$$
\hat{g}_{S}=\frac{\iint g(\beta, \lambda) L\left(\lambda, \beta \mid \mathbf{d}_{\mathbf{n}}\right) \pi(\lambda, \beta) d \lambda d \beta}{\iint L\left(\lambda, \beta \mid \mathbf{d}_{\mathbf{n}}\right) \pi(\lambda, \beta) d \lambda d \beta},
$$




$$
\hat{g}_{L}=-\frac{1}{h} \log \left[\frac{\iint e^{-h g(\beta, \lambda)} L\left(\lambda, \beta \mid \mathbf{d}_{\mathbf{n}}\right) \pi(\lambda, \beta) d \lambda d \beta}{\iint L\left(\lambda, \beta \mid \mathbf{d}_{\mathbf{n}}\right) \pi(\lambda, \beta) d \lambda d \beta}\right]
$$

and

$$
\hat{g}_{E}=\left[\frac{\iint(g(\beta, \lambda))^{-q} L\left(\lambda, \beta \mid \mathbf{d}_{\mathbf{n}}\right) \pi(\lambda, \beta) d \lambda d \beta}{\iint L\left(\lambda, \beta \mid \mathbf{d}_{\mathbf{n}}\right) \pi(\lambda, \beta) d \lambda d \beta}\right]^{-\frac{1}{q}} .
$$

It is not possible to compute (17)-(19) explicitly. Thus we propose MCMC method to find the Bayes estimates for the entropies given in (6) and (7).

\subsection{MCMC Method}

In this subsection, we consider the MCMC method to generate samples from the posterior distributions and then find the Bayes estimates for entropies. The joint posterior distribution given in (16) can be written as

$$
\begin{aligned}
\pi^{*}\left(\beta, \lambda \mid \mathbf{d}_{\mathbf{n}}\right) \propto & \beta^{n+a-1} \lambda^{c+n-1} e^{-\lambda\left(d+r_{n}^{\beta}\right)} \\
& e^{-b \beta} e^{(\beta-1) \sum_{i=1}^{n} \log \left(r_{i}\right)} .
\end{aligned}
$$

From (20) the conditional posterior distribution of $\beta$ given $\lambda$ and data is given by

$$
\pi_{1}^{*}\left(\beta \mid \lambda, \mathbf{d}_{\mathbf{n}}\right) \propto \beta^{n+a-1} e^{-\lambda r_{n}^{\beta}} e^{-\beta\left(b-\sum_{i=1}^{n} \log \left(r_{i}\right)\right)} .
$$

Again from (20), the conditional posterior distribution of $\lambda$ given $\beta$ and data is given by

$$
\pi_{2}^{*}\left(\lambda \mid \beta, \mathbf{d}_{\mathbf{n}}\right) \propto \lambda^{c+n-1} e^{-\lambda\left(d+r_{n}^{\beta}\right)} .
$$

Thus from (22) we can see that conditional posterior distribution of $\lambda$ follows a Gamma distribution with parameters $(n+c)$ and $\left(d+r_{n}^{\beta}\right)$. That is, $\lambda \sim \operatorname{Gamma}\left(n+c, d+r_{n}^{\beta}\right)$. Therefore one can easily generate sample from the posterior distribution of $\lambda$. But it is not possible to generate random variables from the posterior distribution of $\beta$ given in (21) using standard random number generation methods. Hence we use Metropolis-Hasting (M-H) algorithm to generate sample from (21) (see, Chib and Greenberg [20]). Since plot of (21) is similar to a normal plot we take normal proposal density for $\beta$ for the $\mathrm{M}-\mathrm{H}$ algorithm. For updating $\beta$ we have used adaptive MCMC method given in Roberts and Rosenthal [21] to get an optimum acceptance rate (44\%) for the Metropolis sampler.

By setting initial values $\beta^{(0)}$ and $\lambda^{(0)}$, let $\beta^{(t)}$ and $\lambda^{(t)}, t=1,2, \ldots, N$ be the observations generated from (21) and (22) respectively. Then the Bayes estimator of Shannon entropy function given in (6) under SEL, LL and EL, by taking first $m$ iterations as burn-in period, are respectively given by

$$
\begin{gathered}
\hat{H}_{S(M C)}=\frac{1}{N-m} \sum_{t=m+1}^{N} \hat{H}\left(\lambda^{(t)}, \beta^{(t)}\right), \\
\hat{H}_{L(M C)}=-\frac{1}{h} \log \left[\frac{1}{N-m} \sum_{t=m+1}^{N} e^{-h \hat{H}\left(\lambda^{(t)}, \beta^{(t)}\right)}\right]
\end{gathered}
$$

and

$$
\hat{H}_{E(M C)}=\left[\frac{1}{N-m} \sum_{t=m+1}^{N}\left(\hat{H}\left(\lambda^{(t)}, \beta^{(t)}\right)\right)^{-q}\right]^{-\frac{1}{q}},
$$

where

$$
\hat{H}\left(\lambda^{(t)}, \beta^{(t)}\right)=v\left(1-\frac{1}{\beta^{(t)}}\right)-\log \left(\beta^{(t)}\left(\lambda^{(t)}\right)^{\frac{1}{\beta^{(t)}}}\right)+1
$$


Similarly the estimate for Renyi entropy given in (7) under SEL, LL and EL functions are respectively given by

$$
\begin{gathered}
\hat{H}_{S(M C)}(\alpha)=\frac{1}{N-m} \sum_{t=m+1}^{N} \hat{H}_{\alpha}\left(\lambda^{(t)}, \beta^{(t)}\right), \\
\hat{H}_{L(M C)}(\alpha)=-\frac{1}{h} \log \left[\frac{1}{N-m} \sum_{t=m+1}^{N} e^{-h \hat{H}_{\alpha}\left(\lambda^{(t)}, \beta^{(t)}\right)}\right]
\end{gathered}
$$

and

$$
\hat{H}_{E(M C)}(\alpha)=\left[\frac{1}{N-m} \sum_{t=m+1}^{N}\left(\hat{H}_{\alpha}\left(\lambda^{(t)}, \beta^{(t)}\right)\right)^{-q}\right]^{-1 / q}
$$

where

$$
\hat{H}_{\alpha}\left(\lambda^{(t)}, \beta^{(t)}\right)=H_{\alpha}\left(\lambda^{(t)}, \beta^{(t)}\right)
$$

\section{SIMULATION STUDY}

In this section we carry out a simulation study for illustrating the estimation procedures developed in Sections 2 and 3 . First we obtain the MLEs for Shannon entropy and Renyi entropy using (14) and (15). We have obtained the bias and MSE of MLEs for different values of $n$ using 500 simulated samples for different combinations of $\beta$ and $\lambda$ and are given in Tables 1 and 2. For the simulation studies for Bayes estimators we take the hyper parameters for the prior distributions of $\lambda$ and $\beta$ as $a=2, b=2, c=2$ and $d=2$. We have obtained the Bayes estimator for entropy of Weibull distribution using record values under SEL, LL and EL functions using MCMC method. For the MCMC method we do the following

(1) Generate $n$ upper record values from two parameter Weibull distribution with parameters $\lambda$ and $\beta$.

(2) Calculate estimators of entropies using the generated upper-record values using MCMC method as described below.
(a) Start with initial values $\beta^{(0)}$ and $\lambda^{(0)}$.
(b) Set $t=1$.

Table 1 The bias and MSE for maximum likelihood estimator for entropy of Weibull distribution.

\begin{tabular}{cccccc}
\hline $\boldsymbol{n}$ & $\boldsymbol{\lambda}$ & $\boldsymbol{\beta}$ & $\mathbf{H}$ & Bias & MSE \\
\hline 5 & 1 & 2 & 0.30685 & 0.15120 & 0.37001 \\
5 & 1.5 & 2 & 0.32484 & -0.15206 & 0.13575 \\
5 & 2 & 2 & 0.24889 & -0.18819 & 0.09578 \\
5 & 2 & 2.5 & 0.13732 & -0.19030 & 0.10134 \\
5 & 2.5 & 2 & 0.15278 & -0.21610 & 0.09239 \\
6 & 1 & 2 & 0.30685 & 0.14299 & 0.36303 \\
6 & 1.5 & 2 & 0.32484 & -0.14447 & 0.11767 \\
6 & 2 & 2 & 0.24889 & -0.11993 & 0.05973 \\
6 & 2 & 2.5 & 0.13732 & -0.13473 & 0.05981 \\
6 & 2.5 & 2 & 0.15278 & -0.16502 & 0.06212 \\
7 & 1 & 2 & 0.30685 & 0.13766 & 0.36020 \\
7 & 1.5 & 2 & 0.32484 & -0.13625 & 0.11341 \\
7 & 2 & 2 & 0.24889 & -0.07448 & 0.05311 \\
7 & 2 & 2.5 & 0.13732 & -0.09947 & 0.05553 \\
7 & 2.5 & 2 & 0.15278 & -0.12254 & 0.04306 \\
8 & 1 & 2 & 0.30685 & 0.11629 & 0.32744 \\
8 & 1.5 & 2 & 0.32484 & -0.13183 & 0.11810 \\
8 & 2 & 2 & 0.24889 & -0.06559 & 0.04837 \\
8 & 2 & 2.5 & 0.13732 & -0.09507 & 0.04945 \\
8 & 2.5 & 2 & 0.15278 & -0.09617 & 0.03402 \\
\hline
\end{tabular}


Table 2 The bias and MSE for maximum likelihood estimator for Renyi entropy of Weibull distribution.

\begin{tabular}{|c|c|c|c|c|c|c|c|c|}
\hline \multirow[t]{2}{*}{$n$} & \multirow[t]{2}{*}{$\lambda$} & \multirow[t]{2}{*}{$\beta$} & \multicolumn{3}{|c|}{$\alpha=0.5$} & \multicolumn{3}{|c|}{$\alpha=1.5$} \\
\hline & & & $\mathbf{H}$ & Bias & MSE & $\mathbf{H}$ & Bias & MSE \\
\hline 5 & 1 & 2 & 0.693147 & -0.059948 & 0.255098 & 0.117783 & 0.169949 & 0.578651 \\
\hline 5 & 1.5 & 2 & 0.529969 & -0.134760 & 0.116871 & 0.228574 & -0.142179 & 0.163301 \\
\hline 5 & 2 & 2 & 0.406562 & -0.159747 & 0.089425 & 0.170486 & -0.197631 & 0.101358 \\
\hline 5 & 2 & 2.5 & 0.294990 & -0.177099 & 0.089145 & 0.058914 & -0.199679 & 0.108348 \\
\hline 5 & 2.5 & 2 & 0.298591 & -0.190835 & 0.081160 & 0.077009 & -0.225456 & 0.096036 \\
\hline 6 & 1 & 2 & 0.693147 & -0.048178 & 0.210207 & 0.117783 & 0.162723 & 0.564276 \\
\hline 6 & 1.5 & 2 & 0.529969 & -0.093890 & 0.097729 & 0.228574 & -0.089287 & 0.138563 \\
\hline 6 & 2 & 2 & 0.406562 & -0.117676 & 0.064852 & 0.170486 & -0.126982 & 0.064085 \\
\hline 6 & 2 & 2.5 & 0.294990 & -0.111895 & 0.058984 & 0.058914 & -0.142571 & 0.063598 \\
\hline 6 & 2.5 & 2 & 0.298591 & -0.147710 & 0.050369 & 0.077009 & -0.172724 & 0.065074 \\
\hline 7 & 1 & 2 & 0.693147 & 0.038929 & 0.189841 & 0.117783 & 0.150699 & 0.544762 \\
\hline 7 & 1.5 & 2 & 0.529969 & -0.034808 & 0.082219 & 0.228574 & -0.041861 & 0.139014 \\
\hline 7 & 2 & 2 & 0.406562 & -0.088107 & 0.048488 & 0.170486 & -0.102682 & 0.057537 \\
\hline 7 & 2 & 2.5 & 0.294990 & -0.085120 & 0.047481 & 0.058914 & -0.103589 & 0.060667 \\
\hline 7 & 2.5 & 2 & 0.298591 & -0.112599 & 0.039862 & 0.077009 & -0.129293 & 0.045110 \\
\hline 8 & 1 & 2 & 0.693147 & 0.037996 & 0.213258 & 0.117783 & 0.145250 & 0.533804 \\
\hline 8 & 1.5 & 2 & 0.529969 & -0.023618 & 0.080715 & 0.228574 & -0.037239 & 0.140882 \\
\hline 8 & 2 & 2 & 0.406562 & -0.056474 & 0.039593 & 0.170486 & -0.070742 & 0.052154 \\
\hline 8 & 2 & 2.5 & 0.294990 & -0.069971 & 0.040944 & 0.058914 & -0.080985 & 0.053370 \\
\hline 8 & 2.5 & 2 & 0.298591 & -0.088663 & 0.029999 & 0.077009 & -0.101960 & 0.035630 \\
\hline
\end{tabular}

(c) Generate $\lambda^{(t)}$ from Gamma $\left(n+c, d+r_{n}^{\beta^{(t-1)}}\right)$.

(d) Using M-H algorithm, generate $\beta^{(t)}$ from $\pi_{1}^{*}\left(\beta \mid \lambda^{(t)}, \mathbf{d}_{\mathbf{n}}\right)$.

(e) Calculate $\hat{H}\left(\lambda^{(t)}, \beta^{(t)}\right)$ using $(26)$ and $\hat{H}_{\alpha}\left(\lambda^{(t)}, \beta^{(t)}\right)$ using $(30)$.

(f) Set $t=t+1$.

(g) Repeat steps (c) to (f) for $N=50,000$ times.

(h) Calculate the Bayes estimators for Shannon entropy $H(\lambda, \beta)$ using (23) to (25) by taking burn-in period $m=5000$.

(i) Calculate the Bayes estimators for Renyi entropy $H_{\alpha}(\lambda, \beta)$ using (27) to (29) by taking burn-in period $m=5000$.

(3) Repeat the steps 1 and 2 for 500 times.

(4) Calculate the bias and MSE of the estimators.

Repeat the simulation study for $n=5(1) 8$, and for different values of $\beta$ and $\lambda$. The bias and MSE for Bayes estimators for Shannon entropy under SEL, LL and EL functions are given in Table 3. The Bayes estimators for Renyi entropy $H_{\alpha}(\lambda, \beta)$ for $\alpha=0.5$ and $\alpha=1.5$ are obtained. The bias and MSE for the Bayes estimators are given in Tables 4 and 5. From the tables we have the following inference:

(1) The bias and MSE of all estimators decrease when $n$ increases

(2) For Shannon entropy the bias and MSE of Bayes estimator under SEL function, LL function when $h=1$ and EL function when $q=0.5$ are smaller than that of MLE.

(3) For Renyi entropy the bias and MSE of Bayes estimators under EL when $q=0.5$ are smaller than that of MLE.

(4) Among the Bayes estimators, estimators under EL function when $q=0.5$ have least bias and MSE.

\section{ILLUSTRATION USING REAL DATA}

To illustrate the estimation techniques developed in this paper, we consider the real data set given in Lawless [22]. These data are from Nelson [23] concerning the data on time to breakdown of an insulating fluid between electrodes at a voltage of $34 \mathrm{kV}$ (minutes). A model suggested by engineering considerations is that, for a fixed voltage level, time to breakdown has a Weibull distribution (see, Zakerzadeh and Jafari [24]). The 19 times to breakdown are $0.96,4.15,0.19,0.78,8.01,31.75,7.35,6.50,8.27,33.91,32.52,3.16,4.85,2.78,4.67,1.31,12.06,36.71,72.89$. 
Table 3 The bias and MSE for Bayes estimator using MCMC method for Shannon entropy of Weibull distribution.

\begin{tabular}{|c|c|c|c|c|c|c|c|c|c|c|c|c|c|}
\hline \multirow[t]{3}{*}{$n$} & \multirow[t]{3}{*}{$\lambda$} & \multirow[t]{3}{*}{$\beta$} & \multirow[t]{3}{*}{$\mathbf{H}$} & \multicolumn{2}{|c|}{ SEL } & \multicolumn{4}{|c|}{ LL } & \multicolumn{4}{|c|}{ EL } \\
\hline & & & & \multirow[t]{2}{*}{ Bias } & \multirow[t]{2}{*}{ MSE } & \multicolumn{2}{|c|}{$h=-1$} & \multicolumn{2}{|c|}{$h=1$} & \multicolumn{2}{|c|}{$q=-0.5$} & \multicolumn{2}{|c|}{$q=0.5$} \\
\hline & & & & & & Bias & MSE & Bias & MSE & Bias & MSE & Bias & MSE \\
\hline 5 & 1 & 2 & 0.306853 & 0.143080 & 0.096597 & 0.340523 & 0.167842 & -0.145350 & 0.242566 & 0.183398 & 0.054376 & 0.025239 & 0.069449 \\
\hline 5 & 1.5 & 2 & 0.324842 & 0.112276 & 0.037794 & 0.171091 & 0.035764 & -0.095093 & 0.116375 & .128627 & 0.019914 & .008324 & 0.020252 \\
\hline 5 & 2 & 2 & 0.248887 & 0.104140 & 0.019558 & 0.148803 & 0.019410 & 0.034968 & 0.020701 & 0.117607 & 0.011001 & 0.019666 & 0.010130 \\
\hline 5 & 2 & 2.5 & 0.137315 & 0.165508 & 0.014981 & 0.215424 & 0.013658 & 0.143391 & 0.017412 & 197337 & 0.006689 & 0.060376 & 0.005729 \\
\hline 5 & 2.5 & 2 & 0.152780 & 0.121316 & 0.013246 & 0.164007 & 0.014080 & 0.061470 & 0.041449 & 0.150895 & 0.006893 & 0.053233 & 0.006554 \\
\hline 6 & 1 & 2 & 0.306853 & 0.134769 & 0.099796 & 0.250452 & 0.103948 & -0.121101 & 0.142941 & 0.173916 & 0.068909 & 0.002086 & 0.050069 \\
\hline 6 & 1.5 & 2 & 0.324842 & 0.140342 & 0.039032 & 0.190267 & 0.038795 & -0.096762 & 0.120789 & 0.154457 & 0.025543 & 0.020353 & 0.026235 \\
\hline 6 & 2 & 2 & 0.248887 & 0.082657 & 0.015958 & 0.121330 & 0.015852 & -0.032087 & 0.047686 & 0.099380 & 0.009640 & -0.004533 & 0.013504 \\
\hline 6 & 2 & 2.5 & 0.137315 & 0.112191 & 0.016870 & 0.148596 & 0.015433 & 0.108993 & 0.016559 & 0.148833 & 0.007245 & 0.074144 & 0.010614 \\
\hline 6 & 2.5 & 2 & 0.152780 & 0.087899 & 0.012446 & 0.119921 & 0.012482 & 0.052823 & 0.009223 & 0.117893 & 0.005912 & 0.027957 & 0.005149 \\
\hline 7 & 1 & 2 & 0.306853 & 0.121294 & 0.068333 & 0.303026 & 0.070291 & -0.103702 & 0.691284 & 0.221761 & 0.051423 & 0.056752 & 0.069872 \\
\hline 7 & 1.5 & 2 & 0.324842 & 0.134404 & 0.032169 & 0.176695 & 0.030892 & 0.081059 & 0.019577 & 0.147814 & 0.021758 & 0.041127 & 0.023117 \\
\hline 7 & 2 & 2 & 0.248887 & 0.063552 & 0.019593 & 0.091111 & 0.019820 & 0.025151 & 0.008057 & 0.074621 & 0.012175 & -0.015254 & 0.010470 \\
\hline 7 & 2 & 2.5 & 0.137315 & 0.096933 & 0.013217 & 0.124696 & 0.013022 & 0.088383 & 0.011950 & 0.126323 & 0.006319 & 0.051155 & 0.007487 \\
\hline 7 & 2.5 & 2 & 0.152780 & 0.063370 & 0.011832 & 0.085732 & 0.011989 & 0.047954 & 0.007346 & 0.087166 & 0.005458 & 0.007998 & 0.006020 \\
\hline 8 & 1 & 2 & 0.306853 & 0.109351 & 0.074187 & 0.235044 & 0.070451 & -0.061158 & 0.079785 & 0.172108 & 0.050844 & 0.009060 & 0.054591 \\
\hline 8 & 1.5 & 2 & 0.324842 & 0.116412 & 0.032394 & 0.149612 & 0.022140 & 0.077393 & 0.029301 & 0.124510 & 0.024135 & 0.043029 & 0.033992 \\
\hline 8 & 2 & 2 & 0.248887 & 0.037031 & 0.018292 & 0.060380 & 0.018038 & 0.031047 & 0.008966 & 0.051597 & 0.010647 & -0.013535 & 0.010475 \\
\hline 8 & 2 & 2.5 & 0.137315 & 0.091880 & 0.013430 & 0.114333 & 0.013584 & 0.074230 & 0.012085 & 0.116211 & 0.006824 & 0.043945 & 0.006925 \\
\hline 8 & 2.5 & 2 & 0.152780 & 0.070568 & 0.012560 & 0.089709 & 0.012945 & 0.038894 & 0.007220 & 0.087907 & 0.006900 & 0.001881 & 0.006214 \\
\hline
\end{tabular}

Table 4 The bias and MSE for Bayes estimator using MCMC method for Renyi entropy of Weibull distribution when $\alpha=0.5$.

\begin{tabular}{|c|c|c|c|c|c|c|c|c|c|c|c|c|c|}
\hline \multirow[t]{3}{*}{$n$} & \multirow[t]{3}{*}{$\lambda$} & \multirow[t]{3}{*}{$\beta$} & \multirow[t]{3}{*}{$\mathbf{H}$} & \multicolumn{2}{|c|}{ SEL } & \multicolumn{4}{|c|}{ LL } & \multicolumn{4}{|c|}{ EL } \\
\hline & & & & \multirow[t]{2}{*}{ Bias } & \multirow[t]{2}{*}{ MSE } & \multicolumn{2}{|c|}{$h=-1$} & \multicolumn{2}{|c|}{$h=1$} & \multicolumn{2}{|c|}{$q=-0.5$} & \multicolumn{2}{|c|}{$q=0.5$} \\
\hline & & & & & & Bias & MSE & Bias & MSE & Bias & MSE & Bias & MSE \\
\hline 5 & 1 & 2 & 0.693147 & 0.196546 & 0.082497 & 0.813508 & 0.778931 & 0.054065 & 0.078996 & 0.152929 & 0.103351 & 0.022458 & 0.067898 \\
\hline 5 & 1.5 & 2 & 0.529969 & 0.176811 & 0.055391 & 0.798937 & 0.661261 & 0.116578 & 0.035725 & 0.143455 & 0.054033 & 0.040668 & 0.042459 \\
\hline 5 & 2 & 2 & 0.406562 & 0.162975 & 0.060423 & 0.263453 & 0.139603 & 0.140737 & 0.029188 & 0.134038 & 0.036698 & 0.061923 & 0.031100 \\
\hline 5 & 2 & 2.5 & 0.294990 & 0.246542 & 0.084822 & 0.423705 & 0.447057 & 0.178052 & 0.042749 & 0.216017 & 0.099793 & 0.081297 & 0.015073 \\
\hline 5 & 2.5 & 2 & 0.298591 & 0.182706 & 0.089290 & 0.304593 & 0.304736 & 0.167506 & 0.033163 & 0.157428 & 0.040966 & 0.077330 & 0.015939 \\
\hline 6 & 1 & 2 & 0.693147 & 0.177223 & 0.066265 & 0.361536 & 0.542525 & 0.044947 & 0.059022 & 0.140688 & 0.102355 & 0.014939 & 0.050761 \\
\hline 6 & 1.5 & 2 & 0.529969 & 0.182290 & 0.045371 & 0.384255 & 0.511322 & 0.104023 & 0.029926 & 0.136531 & 0.053129 & 0.041075 & 0.036877 \\
\hline 6 & 2 & 2 & 0.406562 & 0.145335 & 0.047646 & 0.199118 & 0.062987 & 0.094076 & 0.018461 & 0.120119 & 0.030960 & 0.022167 & 0.023291 \\
\hline 6 & 2 & 2.5 & 0.294990 & 0.167407 & 0.047461 & 0.215544 & 0.071396 & 0.166097 & 0.039095 & 0.146616 & 0.036630 & 0.070173 & 0.022421 \\
\hline 6 & 2.5 & 2 & 0.298591 & 0.136886 & 0.045278 & 0.176971 & 0.050306 & 0.114437 & 0.022314 & 0.116736 & 0.026724 & 0.039207 & 0.013904 \\
\hline 7 & 1 & 2 & 0.693147 & 0.181780 & 0.058457 & 0.343809 & 0.246411 & 0.118695 & 0.048293 & 0.132774 & 0.085692 & 0.016012 & 0.040022 \\
\hline 7 & 1.5 & 2 & 0.529969 & 0.168940 & 0.039860 & 0.334644 & 0.338679 & 0.120777 & 0.027189 & 0.126735 & 0.045108 & 0.035957 & 0.031654 \\
\hline 7 & 2 & 2 & 0.406562 & 0.096236 & 0.040584 & 0.132437 & 0.043320 & 0.076677 & 0.015079 & 0.077070 & 0.025269 & 0.012859 & 0.018218 \\
\hline 7 & 2 & 2.5 & 0.294990 & 0.135825 & 0.033213 & 0.166573 & 0.041962 & 0.116869 & 0.025366 & 0.117778 & 0.025622 & 0.057641 & 0.016409 \\
\hline 7 & 2.5 & 2 & 0.298591 & 0.089041 & 0.022729 & 0.113852 & 0.026272 & 0.076116 & 0.011697 & 0.083620 & 0.017741 & 0.008698 & 0.012332 \\
\hline 8 & 1 & 2 & 0.693147 & 0.159678 & 0.049626 & 0.250205 & 0.196829 & 0.055757 & 0.044402 & 0.129939 & 0.076439 & 0.009386 & 0.032933 \\
\hline 8 & 1.5 & 2 & 0.529969 & 0.127840 & 0.030039 & 0.162985 & 0.057665 & 0.086131 & 0.018567 & 0.109171 & 0.041364 & 0.027335 & 0.024476 \\
\hline 8 & 2 & 2 & 0.406562 & 0.064855 & 0.017210 & 0.089315 & 0.025790 & 0.064308 & 0.014565 & 0.048934 & 0.018451 & 0.009570 & 0.016832 \\
\hline 8 & 2 & 2.5 & 0.294990 & 0.118928 & 0.023678 & 0.143125 & 0.035012 & 0.111415 & 0.022639 & 0.103322 & 0.023135 & 0.050477 & 0.014464 \\
\hline 8 & 2.5 & 2 & 0.298591 & 0.094234 & 0.013884 & 0.104579 & 0.025505 & 0.056222 & 0.011352 & 0.079402 & 0.016641 & 0.000468 & 0.010885 \\
\hline
\end{tabular}

Thus the six upper record values obtained from the above data are 
Table 5 The bias and MSE for Bayes estimator using MCMC method for Renyi entropy of Weibull distribution when $\alpha=1.5$.

\begin{tabular}{|c|c|c|c|c|c|c|c|c|c|c|c|c|c|}
\hline \multirow[t]{3}{*}{$n$} & \multirow[t]{3}{*}{$\lambda$} & \multirow[t]{3}{*}{$\beta$} & \multirow[t]{3}{*}{$\mathbf{H}$} & \multicolumn{2}{|c|}{ SEL } & \multicolumn{4}{|c|}{ LL } & \multicolumn{4}{|c|}{ EL } \\
\hline & & & & \multirow[t]{2}{*}{ Bias } & \multirow[t]{2}{*}{ MSE } & \multicolumn{2}{|c|}{$h=-1$} & \multicolumn{2}{|c|}{$h=1$} & \multicolumn{2}{|c|}{$q=-0.5$} & \multicolumn{2}{|c|}{$q=0.5$} \\
\hline & & & & & & Bias & MSE & Bias & MSE & Bias & MSE & Bias & MSE \\
\hline 5 & 1 & 2 & 0.117783 & 0.143785 & 0.214935 & 0.809917 & 0.830768 & -0.812705 & 0.915072 & 0.153166 & 0.057908 & 0.093631 & 0.040360 \\
\hline 5 & 1.5 & 2 & 0.228574 & 0.097911 & 0.048286 & 0.152334 & 0.055674 & -0.599903 & 0.696059 & 0.162594 & 0.037728 & 0.081120 & 0.023191 \\
\hline 5 & 2 & 2 & 0.170486 & 0.054085 & 0.022127 & 0.107969 & 0.038909 & -0.789021 & 0.743881 & 0.124505 & 0.027592 & 0.010485 & 0.007134 \\
\hline 5 & 2 & 2.5 & 0.058914 & 0.127366 & 0.023164 & 0.183583 & 0.069983 & -0.227253 & 0.828956 & 0.187259 & 0.047305 & 0.106829 & 0.004858 \\
\hline 5 & 2.5 & 2 & 0.077009 & 0.113595 & 0.018904 & 0.169285 & 0.058878 & -0.518612 & 0.647417 & 0.179826 & 0.053159 & 0.061674 & 0.007146 \\
\hline 6 & 1 & 2 & 0.117783 & 0.123926 & 0.161183 & 0.674674 & 0.691893 & -0.653557 & 0.855418 & 0.208283 & 0.057167 & 0.042790 & 0.042846 \\
\hline 6 & 1.5 & 2 & 0.228574 & 0.080413 & 0.046841 & 0.146626 & 0.045126 & -0.426703 & 0.577858 & 0.156055 & 0.036695 & 0.067811 & 0.018417 \\
\hline 6 & 2 & 2 & 0.170486 & 0.031606 & 0.020470 & 0.096560 & 0.018792 & -0.388842 & 0.612311 & 0.097146 & 0.017333 & 0.006862 & 0.007919 \\
\hline 6 & 2 & 2.5 & 0.058914 & 0.087448 & 0.019065 & 0.132682 & 0.045161 & -0.152802 & 0.776322 & 0.166613 & 0.035097 & 0.087896 & 0.004021 \\
\hline 6 & 2.5 & 2 & 0.077009 & 0.077770 & 0.013118 & 0.108067 & 0.032541 & -0.196939 & 0.589683 & 0.141192 & 0.032973 & 0.049119 & 0.005657 \\
\hline 7 & 1 & 2 & 0.117783 & 0.127652 & 0.113003 & 0.555421 & 0.517746 & -0.492061 & 0.750442 & 0.147221 & 0.046980 & 0.032156 & 0.041178 \\
\hline 7 & 1.5 & 2 & 0.228574 & 0.060234 & 0.030591 & 0.122757 & 0.036892 & -0.373434 & 0.355197 & 0.125840 & 0.022963 & 0.040831 & 0.015223 \\
\hline 7 & 2 & 2 & 0.170486 & 0.035268 & 0.017766 & 0.083967 & 0.018368 & -0.186198 & 0.572364 & 0.094418 & 0.012888 & 0.003683 & 0.006916 \\
\hline 7 & 2 & 2.5 & 0.058914 & 0.076822 & 0.017810 & 0.128533 & 0.028193 & -0.147492 & 0.577554 & 0.167304 & 0.028828 & 0.063494 & 0.004193 \\
\hline 7 & 2.5 & 2 & 0.077009 & 0.058212 & 0.012529 & 0.082072 & 0.013411 & 0.089488 & 0.216347 & 0.121062 & 0.023789 & 0.038882 & 0.002229 \\
\hline 8 & 1 & 2 & 0.117783 & 0.105772 & 0.109842 & 0.314116 & 0.428191 & -0.465304 & 0.547167 & 0.133842 & 0.039836 & 0.025446 & 0.050438 \\
\hline 8 & 1.5 & 2 & 0.228574 & 0.025821 & 0.029142 & 0.113412 & 0.034319 & -0.342524 & 0.320062 & 0.110418 & 0.017284 & 0.029241 & 0.014719 \\
\hline 8 & 2 & 2 & 0.170486 & 0.020583 & 0.016766 & 0.087848 & 0.017410 & 0.013301 & 0.059215 & 0.086408 & 0.010146 & 0.001713 & 0.005958 \\
\hline 8 & 2 & 2.5 & 0.058914 & 0.071074 & 0.013908 & 0.096153 & 0.013318 & -0.012665 & 0.251670 & 0.118776 & 0.024196 & 0.056404 & 0.003458 \\
\hline 8 & 2.5 & 2 & 0.077009 & 0.047979 & 0.011430 & 0.066928 & 0.011708 & -0.066936 & 0.137418 & 0.103945 & 0.023205 & 0.026421 & 0.002190 \\
\hline
\end{tabular}

We have obtained the estimators for Shannon entropy and Renyi entropy and are given below. For the Bayes estimation we took the noninformative priors for $\lambda$ and $\beta$ and are obtained when $a=0, b=0, c=0$ and $d=0$. For MCMC method we took number of iterations as $N=50,000$ and first $m=5000$ iterations as burn-in period. The estimators for Shannon entropy using different methods are given below.

\begin{tabular}{ccccc}
\hline \multicolumn{2}{c}{ MLE } & & $\mathbf{2 . 4 2 3 7}$ \\
\hline Bayes estimator & \multicolumn{2}{c}{ SEL } & & $\mathbf{2 . 1 5 6 3}$ \\
\hline & LL & $h=-1$ & & 2.1945 \\
& & $h=1$ & & 1.9874 \\
& EL $\quad$ & $\mathrm{q}=-0.5$ & & 1.9879 \\
& & $\mathrm{q}=0.5$ & 1.8241 \\
\hline
\end{tabular}

Also the estimators for Renyi entropy using different methods are given below.

\begin{tabular}{|c|c|c|c|c|}
\hline \multicolumn{3}{|c|}{$\alpha$} & 0.5 & 1.5 \\
\hline \multicolumn{3}{|c|}{ MLE } & 3.0179 & 2.0983 \\
\hline \multirow[t]{5}{*}{ Bayes estimator } & & & 2.8231 & 1.8923 \\
\hline & LL & $h=-1$ & 2.6142 & 1.8756 \\
\hline & & $h=1$ & 2.6987 & 1.9982 \\
\hline & EL & $q=-0.5$ & 2.6574 & 1.9475 \\
\hline & & $q=0.5$ & 2.7462 & 1.9526 \\
\hline
\end{tabular}

\section{CONCLUSION}

In this work, we have considered the problem of estimation of entropy for a two parameter Weibull distribution using record values. The maximum likelihood estimation and Bayesian estimation have been applied to obtain estimators for Shannon and Renyi entropies. For obtaining the Bayes estimates, MCMC method has been applied. Based on the simulation study we have the following conclusions. The 
bias and MSE of all estimators decrease when $n$ increases. The Bayes estimators for both Shannon entropy and Renyi entropy using MCMC method perform better than corresponding MLEs. Among the Bayes estimators, estimators under entropy loss function perform better.

\section{CONFLICTS OF INTEREST}

The authors declare that they have no conflicts of interest.

\section{AUTHORS' CONTRIBUTIONS}

The first author developed the methodology and editing of the paper while the second author performed the writing up of the material and conducting the numerical simulation.

\section{ACKNOWLEDGMENTS}

The authors are thankful to the referees and editor for their careful reading and comments.

\section{REFERENCES}

1. K.N. Chandler, J. R. Stat. Soc. Ser. B. 14 (1952), 220-228.

2. M. Ahsanullah, Record Statistics, Nova Science Publishers, New York, NY, USA, 1995.

3. B.C. Arnold, N. Balakrishnan, H.N. Nagaraja, Records, Wiley, New York, NY, USA, 1998.

4. U. Kamps, Commun. Stat. Theory Methods. 23 (1994), 2101-2112.

5. N. Balakrishnan, P.S. Chan, Stat. Probab. Lett. 39 (1998), 73-80.

6. M. Raqab, J. Stat. Plan. Inference. 104 (2002), 339-350.

7. K.S. Sultan, M.E. Moshref, A. Childs, J. Appl. Stat. Sci. 11 (2002), 143-156.

8. J. Ahmadi, M. Doostparast, Stat. Pap. 47 (2006), 373-392.

9. J.I. Seo, H.J. Lee, S.B. Kang, J. Korean Data Inf. Sci. 23 (2012), 1249-1257.

10. C.E. Shannon, Bell Syst. Tech. J. 27 (1948), 379-432.

11. A. Renyi, in: Proceeding of Fourth Berkeley Symposium on Mathematical Statistics and Probability, University of California Press, Berkeley, CA, USA, 1961, pp. 547-561.

12. S.B. Kang, Y.S. Cho, J.T. Han, J. Kim, Entropy. 14 (2012), 161-173.

13. Y. Cho, H. Sun, K. Lee, Entropy. 16 (2014), 3655-3669.

14. S. Baratpour, J. Ahmadi, N.R. Arghami, Stat. Pap. 48 (2007), 197-213.

15. M. Chacko, P.S. Ashs, J. Indian Soc. Probab. Stat. 19 (2018), 79-96.

16. D.N.P. Murthy, M. Xie, R. Jiang, Weibull Models, Wiley, Hoboken, NY, USA, 2004.

17. Z.S. Ye, Y. Hong, Y. Xie, Ann. Appl. Stat. 7 (2013), 2249-2271.

18. E. Cramer, C. Bagh, Commun. Stat. Theory Methods. 40 (2011), 2511-2527.

19. Y. Cho, H. Sun, K. Lee, Entropy. 17 (2015), 102-122.

20. S. Chib, E. Greenberg, Am. Stat. 49 (1995), 327-335.

21. G.O. Roberts, J.S. Rosenthal, J. Comput. Graph. Stat. 18 (2009), 349-367.

22. J.F. Lawless, Statistical Models and Methods for Lifetime Data, Wiley-Interscience, New York, NY, USA, 2003.

23. W. Nelson, Applied Life Data Analysis, Wiley, New York, NY, USA, 1982.

24. H. Zakerzadeh, A.A. Jafari, Stat. Methods Appl. 24 (2015), 25-40. 